\title{
ORES: a chassis dynamometer for off-road vehicles
}

\author{
Husain Kanchwala* \\ Warwick Manufacturing Group, University of Warwick, Coventry CV4 7AL, UK
}

Received: 16 June 2020 / Accepted: 20 January 2021

\begin{abstract}
Off-Road Environment Simulator (ORES) is a Real-time Hardware-in-the-Loop (RT-HIL) platform to simulate the dynamic response of off-road vehicles. This paper primarily focuses on the vehicle model development and validation using both field and rig testing using the ORES platform. Off-road vehicles are capable of operating on bumpy terrains where they are subjected to different resistive wheel torques due to non-unique ground friction conditions and wheel loads. If the powertrain torque output is not distributed in accordance with the resistive wheel torques, it may lead to transmission windup resulting in premature failure of various driveline components. In this study, the vehicle is driven over discrete bumps both in rig simulation and field trial. Different terrain enveloping models were evaluated namely the single point, radial-spring contact model and two-point follower (using circular and sinusoidal basis). These models were evaluated against the measured wheel acceleration responses. The two-point follower with sinusoidal basis strongly correlates with the measured responses and the ground excitations so obtained were used as inputs to a seven degree-of-freedom vehicle ride model. Ride model calculates the wheel loads and is eventually integrated with longitudinal dynamics, tire, driveline and test-rig models. Vehicle axle acceleration, wheel speed and drive torque responses are measured for validating the simulation results against field and rig trials. The field responses matches fairly well which validates the suitability of the proposed modeling approach.
\end{abstract}

Keywords: RT-HIL systems / driveline / terrain modeling / ride dynamics / longitudinal dynamics

\section{Introduction}

All-wheel-drive (AWD) vehicles are designed to be capable of operating in off-road environments. The vehicle is generally driven on these bumpy terrains at low-speeds and high drive torques. At such low frequencies, the driveline dynamics are strongly connected with the longitudinal and pitch motions of vehicle body [1]. This requires accurate modeling of body and suspension dynamics [2-4]. Undesired vibrations are introduced in the driveline by tires which act as an interconnection between road and driveline as external rotating masses [5]. It is important to conduct an in-depth investigation of the effect of tire-terrain interaction on driveline oscillations [6]. In addition to the vertical ride dynamics, longitudinal tire slip calculation is also critical [7]. The resulting thrust force obtained from the vertical dynamics and the longitudinal slip dynamics can be effectively used to simulate several prominent driveline effects like 'Shuffle' (2-8 Hz) and 'Judder' (7-20 Hz) [8].

These off-road conditions are hard to replicate in a repetitive fashion in field-testing using a prototype. To replicate and characterize the off-road

\footnotetext{
* e-mail: husain.kanchwala@warwick.ac.uk
}

vehicle performance, an Off-Road Environment Simulator (ORES) vehicle development platform was developed at the Off-Road Dynamics facility at Cranfield University. The primary purpose of this HIL platform is to simulate the dynamic response of the vehicle to such transient torque inputs in a reliable and repeatable lab-based environment. This platform will substantially reduce the transmission development time and field testing expenditure [9]. The vehicle used for this study is an AWD Range Rover Sport [10]. The dynamics of the tire when excited by short wavelength road profiles, like a bump of cleat, is extremely complex [11]. For these discontinuous short-wavelength ground excitations, terrain enveloping plays a significant role in determining effective ground excitation. A terrain-enveloping model is used to get an effective terrain experienced at the wheel axle. The vehicle is mounted on the ORES platform which simulates the vehicle response in terms of the resistances it experiences at the tire-terrain interface as it moves over a discrete bump.

The paper is organized as follows: ORES platform is discussed in Section 2. The vehicle model is described in Section 3. Next the terrain modelling is discussed in Section 4. Terrain data acquisition, filtering and implementation for test simulations is discussed in detail. 


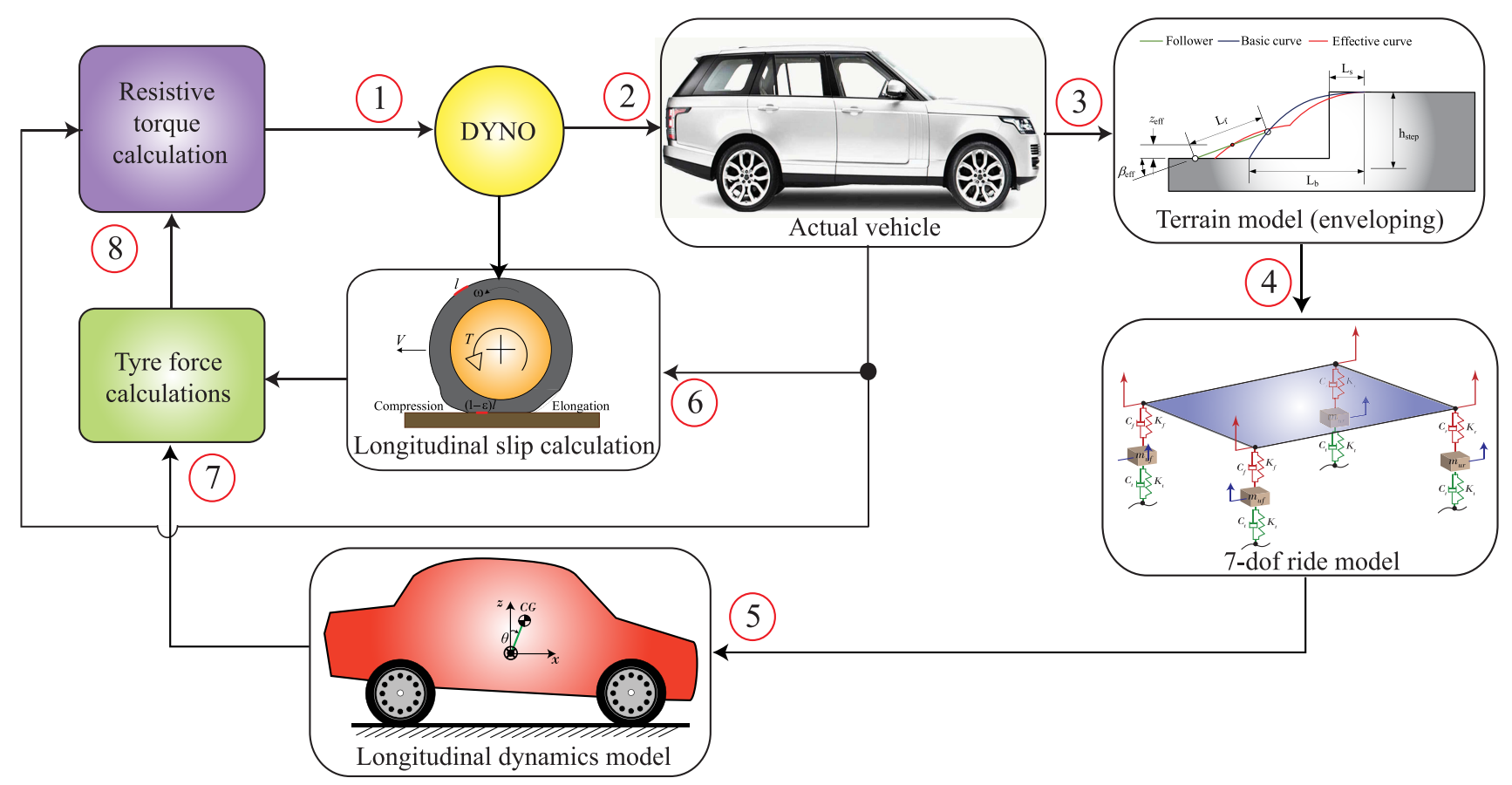

Fig. 1. Working principle of the ORES platform.

Different terrain-wheel enveloping models for calculating the vertical wheel accelerations were developed namely, single-point contact, radial-spring contact model and twopoint follwer enveloping tire model in Section 5. The field and rig test simulation results for different tire models are shown in Section 8. Conclusions of the work are summarised in Section 9. Finally the future directions which can be explored to extend this work are discussed in Section 10.

\section{ORES: off-road environment simulator}

The aim of the ORES platform is to replace a significant portion of the off-road testing by providing a virtual test environment using a RT-HIL test bench.

In a vehicle, the ride quality experienced by a driver is affected by both suspension response and driveline oscillations. The latter are indirectly affected by the former, but in the present test setup the suspension (and road surface) are simulated externally while the driveline effects are studied in the physical vehicle. To this end, the physical vehicle is held immobile while its wheels are driven, using the accelerator pedal in the vehicle, by a person in the car. From outside, the wheels are given resistive torques using dynamometers. The rest of the activity for computing what those resistive torques should be is outlined in the flowchart as shown in Figure 1.

It comprises of four elements: A physical interface to the vehicle, a virtual test terrain/environment, a virtual model of the test vehicle and a virtual model of the tire/terrain interface. These four elements interact together to form the ORES modelling environment. The core working principle behind the rig is to apply the computed resistive torques on the vehicle hub. Different steps involved are described below.

To begin with the resistive torque inputs are given to the four individual dynamometer (abbreviated as dyno, one for each wheel) as shown in step (1). The dyno applies the torques to the vehicle by means of wheel-hub shaft couplings in step (2). The vehicle is driven at a predefined speed by applying a throttle input by the driver. The vehicle remains stationary and only the drive axles rotate. In addition, the effect of the variation of the vertical loads on the wheels is captured by means of varying resistive torque inputs coming from the dynamometer on each individual wheel. The wheel speeds, vehicle speed and torques are measured by means of a speed encoder and wheel torque transducer. The details about the rig construction, sensor instrumentation, actuator and data acquisition is given in [12]. The vehicle position is obtained from the measurement of vehicle speed and is used to calculate the effective terrain by a terrain enveloping model in step (3), simultaneously the recorded wheel speeds are used for longitudinal wheel slip calculation in step 6). The terrain enveloping model computes the effective ground displacements which are in-turn used by the seven degrees-of-freedom (dof) vehicle ride model to calculate the normal loads on each wheel as the vehicle traverses over the bumpy terrain. These wheel loads are used as inputs to the longitudinal dynamics model in step (5). Finally the longitudinal dynamics model along with the wheel slip calculations coming from the tire model is used to calculate the tire forces and the vehicle acceleration in step (7). These forces are transformed to the resistive torques by taking the actuator saturation and traction limit into account in step (8). To ensure that these resistive tyre forces are applied to the power train, a mechanical 


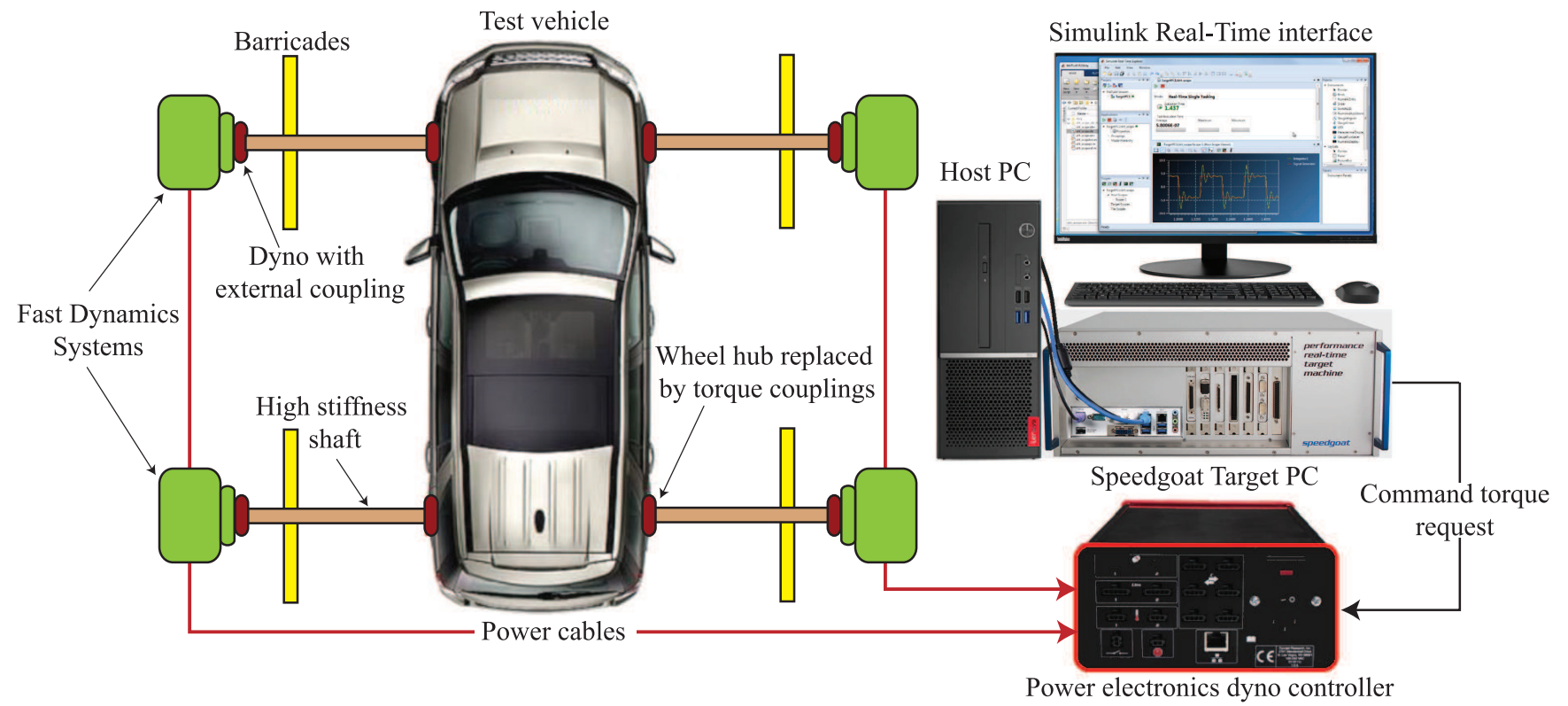

Fig. 2. ORES rig schematics.

interface must exist between the power train and the force generation device which is the dynamometer as shown in step (1).

The schematic diagram of the test platform is shown in Figure 2. Each individual dyno is coupled to the wheel hub using a high tensile shaft. These dynos are driven by resistive torque command from power electronics controller.

Generally commercial dynamometers employ a drum roller as an interface between the dyno-motor and vehicle [13]. This requires knowledge of tire/drum interface to be taken into account. However during a test if the friction limit for tire/drum interface is exceeded before that of the theoretical tire/terrain interface then the rig performance would be compromised. This direct hub coupling approach in ORES breaks new ground in this direction as no physical slip could occur and any tire-road interface can be simulated by always maintaining the rig performance.

In the ORES test rig, the resistive torque request which emulates the resistance experienced by the vehicle going over a bumpy terrain is calculated by the simulation model of the vehicle as discussed in Figure 1.

The vehicle model is developed in MATLAB/Simulink platform. The model has to work in real-time so it is loaded to the Speedgoat RT target PC which is connected to the host PC (in which the model is loaded) using Simulink RT [14]. The resistive torques computed by the vehicle model are then fed to the power electronics controller which ensures that the torques are applied to the four dynos in real-time. The wheel axles rotate in accordance with the throttle request by the driver.

The vehicle remains stationary and the resistive torque captures the effect of vehicle suspension loads as it traverses on the virtual terrain modeled by means of a tire-terrain model. The key elements of the vehicle model are discussed below.

\section{Vehicle model}

The vehicle model consists of a seven degrees-of-freedom ride model that incorporates roll, pitch and jounce motions to simulate the ride behaviour. In addition, a longitudinal dynamics model along with the Magic Formula tire model for generating the longitudinal force is developed [15]. Two versions of the vehicle models were developed. The first one is an offline model which was used for field tests. This model has in-built driveline consisting of engine and transmission model. The second model is developed for the rig simulation where the transmission model is replaced by actual vehicle driveline on the dynamometer. The vehicle ride and longitudinal dynamics models are discussed below.

\subsection{Vehicle ride dynamics}

The ride characteristics primarily depend on the vehicle suspension and tire parameters mainly stiffness; and damping. This model is used to calculate the dynamic tire load and not the frequency response analysis of the system. The scope of the ride analysis is limited merely to get the wheel loads.

The vehicle ride dynamics can be described by the seven degree-of-freedom (dof) model as shown in Figure 3.

The vehicle ride model has 4 dofs corresponding to wheel jounce $z_{1}$ through $z_{4}$ and 3 additional dofs for bounce $z$, roll $\phi$ and pitch $\theta$ respectively. The ground displacements corresponding to the front and rear left/right wheel contact points are given by $u_{1}$ through $u_{4}$ respectively.

The vertical dynamics of the vehicle can be represented by the following set of equations:

$$
\begin{aligned}
M_{s} \ddot{z}= & -K_{f} z_{f l}-K_{f} z_{f r}-K_{r} z_{r l}-K_{r} z_{r r} \\
& -C_{f} \dot{z}_{f l}-C_{f} \dot{z}_{f r}-C_{r} \dot{z}_{r l}-C_{r} \dot{z}_{r r}-M_{s} g .
\end{aligned}
$$




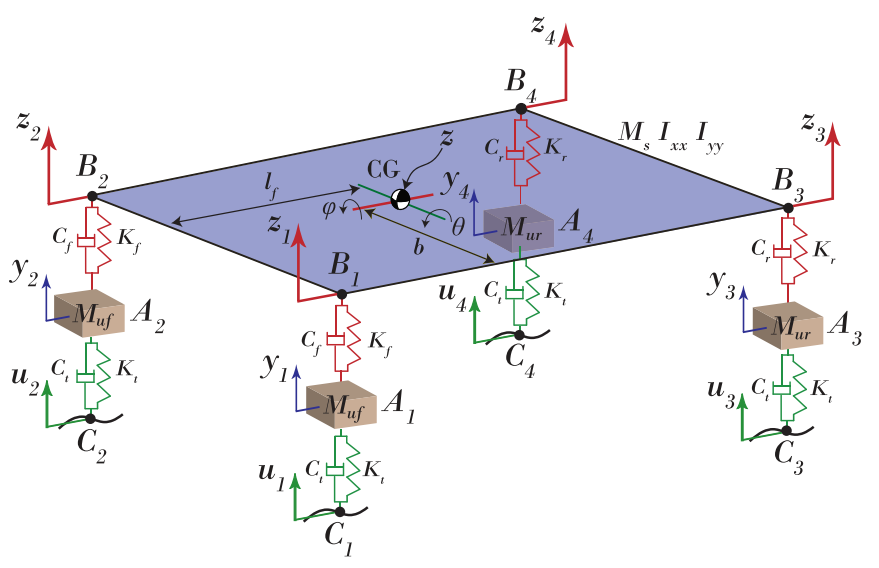

Fig. 3. Vertical dynamics of the vehicle body.

$$
\begin{aligned}
I_{x x} \ddot{\theta}= & K_{f} l_{f} z_{f l}+K_{f} l_{f} z_{f r}-K_{r} l_{r} z_{r l}-K_{r} l_{r} z_{r r} \\
& +C_{f} l_{f} \dot{z}_{f l}+C_{f} l_{f} \dot{z}_{f r}-C_{r} l_{r} \dot{z}_{r l}-C_{r} l_{r} \dot{z}_{r r} . \\
I_{y y} \ddot{\phi}= & -K_{f} b z_{f l}-K_{r} b z_{r l}+K_{f} b z_{f r}+K_{r} b z_{r r} \\
& -C_{f} b \dot{z}_{f l}-C_{r} b \dot{z}_{r l}+C_{f} b \dot{z}_{f r}+C_{r} b \dot{z}_{r r} .
\end{aligned}
$$

$$
\begin{aligned}
& M_{u f} \ddot{z}_{1}=K_{f} z_{f l}+C_{f} \dot{z}_{f l}-K_{t} z_{t f l}-C_{t} \dot{z}_{t f l}-M_{u f} g, \\
& M_{u f} \ddot{z}_{2}=K_{f} z_{f r}+C_{f} \dot{z}_{f r}-K_{t} z_{t f r}-C_{t} \dot{z}_{t f r}-M_{u f} g, \\
& M_{u r} \ddot{z}_{3}=K_{r} z_{r l}+C_{f} \dot{z}_{r l}-K_{t} z_{t r l}-C_{t} \dot{z}_{t r l}-M_{u r} g, \\
& M_{u r} \ddot{z}_{4}=K_{r} z_{r r}+C_{f} \dot{z}_{r r}-K_{t} z_{t r r}-C_{t} \dot{z}_{t r r}-M_{u r} g .
\end{aligned}
$$

In equation (4) the effective suspension and wheel travel is given by

$$
\begin{gathered}
z_{f l}=z_{1}-y_{1}, z_{f r}=z_{2}-y_{2}, z_{r l}=z_{3}-y_{3}, z_{r r}=z_{4}-y_{4}, \\
z_{t f l}=y_{1}-u_{1}, z_{t f r}=y_{2}-u_{2}, z_{t r l}=y_{3}-u_{3}, z_{t r r}=y_{4}-u_{4},
\end{gathered}
$$

where $\quad z_{1}=z-l_{f} \theta+b \phi, \quad z_{2}=z-l_{f} \theta-b \phi$

$$
z_{3}=z+l_{r} \theta+b \phi, \quad z_{4}=z+l_{r} \theta-b \phi .
$$

From the tire deflections the dynamic tire load (say frontleft) is obtained as

$$
F_{z 1}=-\left(K_{t} z_{t f l}+C_{t} \dot{z}_{t f l}\right) \cos \beta_{f l}
$$

In equation (5), $\beta_{f l}$ is the effective plane angle obtained from the terrain enveloping model in Section 4. Next the longitudinal dynamics model is discussed.

\subsection{Vehicle longitudinal dynamics}

The vehicle longitudinal model calculates the position, velocity and acceleration of the vehicle as it traverses the undulated terrain. This is required because when the simulation is performed in the time domain, the vehicle position with respect to the road is used as an input for applying the vertical excitation coming from the ground depending on the obstacle location. The velocity is required to be fed into the terrain model as it controls the

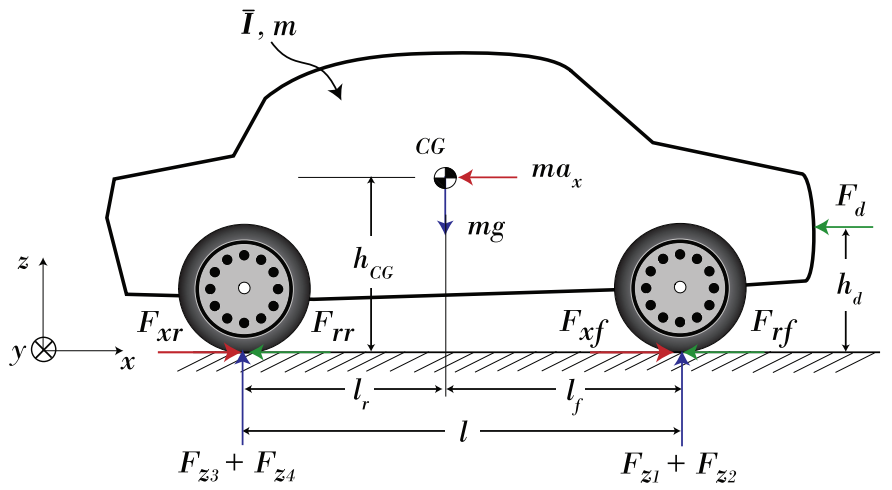

Fig. 4. Longitudinal dynamics of the vehicle.

speed at which an unevenness would hit the tire. Finally, the acceleration term is fed into the ride model in order to calculate the longitudinal weight transfer which cause the pitching motion of the vehicle body. In addition to these responses, the vehicle longitudinal dynamics model also calculates longitudinal propulsive force which is inturn used to compute the resistive torques. The resistive torques are eventually given as inputs to the rig via the power-electronics controller (see Fig. 2). From the free body diagram of the vehicle in Figure 4 we obtain

$$
m a_{x}=\sum_{i=1}^{4}\left(F_{x i}-F_{r i}\right)-F_{d}-F_{b}
$$

$$
\text { where } F_{r i}=f F_{z i} ; \quad \text { and } F_{d}=\frac{1}{2} C_{d} \rho A V^{2} \text {. }
$$

where $i \in 1-4$ for front left/right and rear left/right respectively; $F_{b}$ is the total bump resistance encountered by tires while going over an obstacle (see Sect. 4 ); $f$ and $C_{d}$ are the rolling resistance and drag coefficients; $\rho$ is the density of air; $A$ is the car frontal area and $V$ is the longitudinal velocity. The values of the parameters $f, C_{d}$ and $A$ were made available by the vehicle manufacturer. The longitudinal force $F_{x i}$ generated by the tire is computed using the tire model as described in Section 5 .

\section{Terrain modeling}

Vehicle performance on an off-road terrain to great extent depends on the manner in which it interacts with the terrain [16]. Hence, it is important to have an understanding of vehicle-terrain interaction mechanics for analysing the vertical dynamics of a vehicle [17]. Two main methods of terrain modelling are: online and offline identification [18]. Online methods rely on sensors or cameras to detect road geometry data which is then fed into highperformance CPU to process real-time data and estimate terrain parameters. This method has limited applications due the high cost involved, for this reason an offline terrain modeling method is used in this manuscript. 


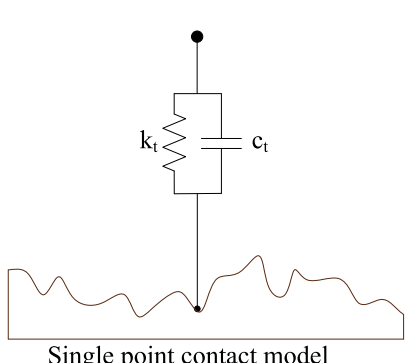

(a)

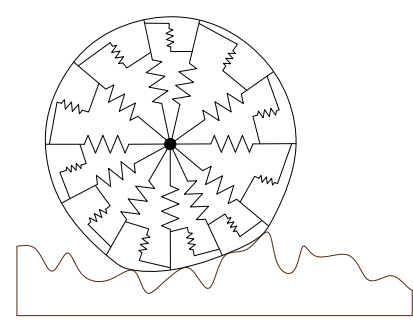

Radial-interrelated spring model

(e)

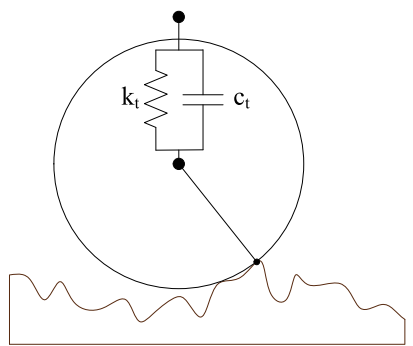

Roller contact model

(b)

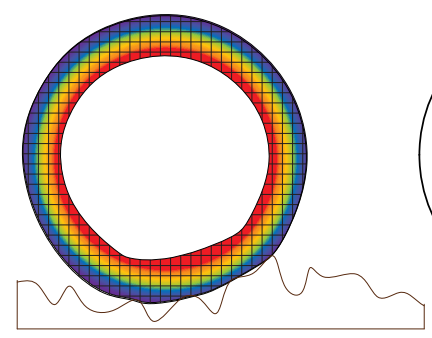

Finite element model

(f)

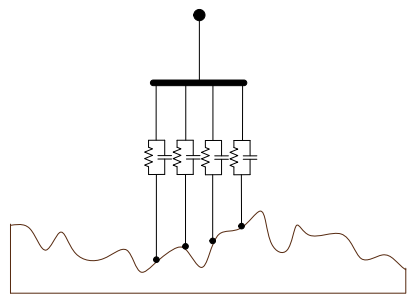

Fixed footprint model

(c)

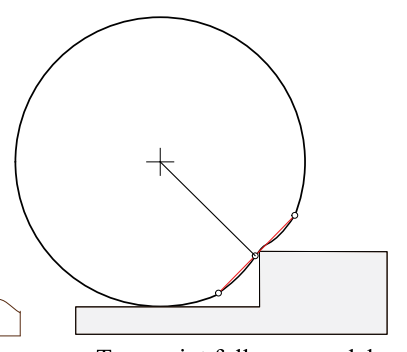

(g)

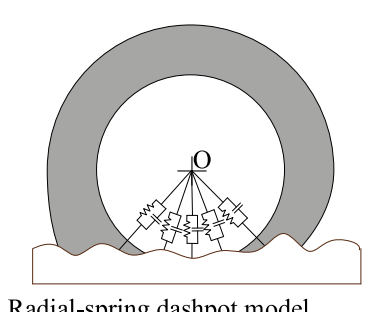

(d)

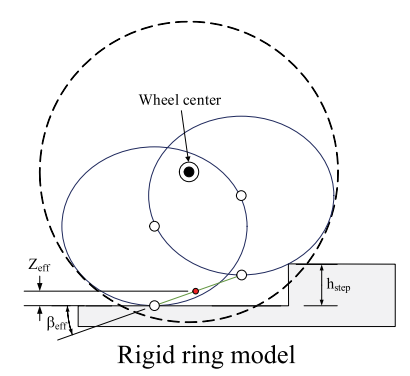

(h)

Fig. 5. Different tire terrain filtering models.

For road wavelengths greater than $1.5 \mathrm{~m}$, the geometry of the road surface can be directly used as input excitation [7]. For short wavelengths, it is necessary to include the geometric filtering due to finite tire dimensions which smoothens the sharp edges of unevenness [19]. If no terrain filtering is used for these short wavelength excitations like a cleat obstacle, the vertical accelerations experienced by the wheel are unrealistic. Different types of tire models can be used in order to consider the effect of terrain filtering as shown in Figure 5.

\subsection{Tire-terrain enveloping models}

In literature, several authors have provided a detailed overview of various terrain enveloping models [20-22]. These models can be broadly classified as:

1. Point contact models [23]

2. Roller contact models [24]

3. Fixed footprint models [25]

4. Radial-spring models [26]

5. Finite element models [27]

6. Rigid ring models [28]

7. Multi-body models [29].

For the purpose of selecting a suitable terrain enveloping model, initial simulation studies were performed to compare various models. From the above different class of models, the single point contact, two-point follower and radial-spring tire models were considered in this study. Other models, for instance, radial-interrelated and multibody models are computationally complex, footprint models require extra parameters like the footprint length which changes with bump size, speed and load, and finite element models are unsuitable for real-time applications. For real-time implementation, computational time is a

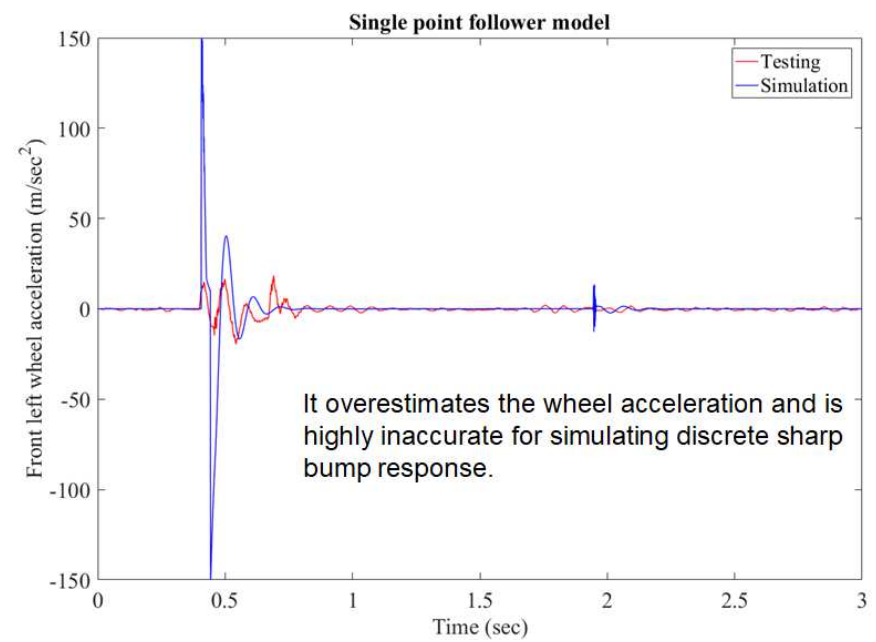

Fig. 6. Single point follower model fitting against test responses.

major limitation and the computation time should be at least 20 times faster than the rig response time in order to get meaningful rig simulation results. Researchers in general have widely studied the computational times for various models [30]. These three models were considered because to their suitability for the real-time implementation.

For the initial validation purpose, the vehicle is driven over a rectangular bump $(100 \times 70 \mathrm{~mm})$ at $30 \mathrm{kmph}$. An accelerometer is mounted on the front-left axle and the vertical accelerations were recorded. The field test measurements were compared against simulations results of the above three models. To start with it has been found that single-point contact model greatly overestimates the measured responses as shown in Figure 6. 


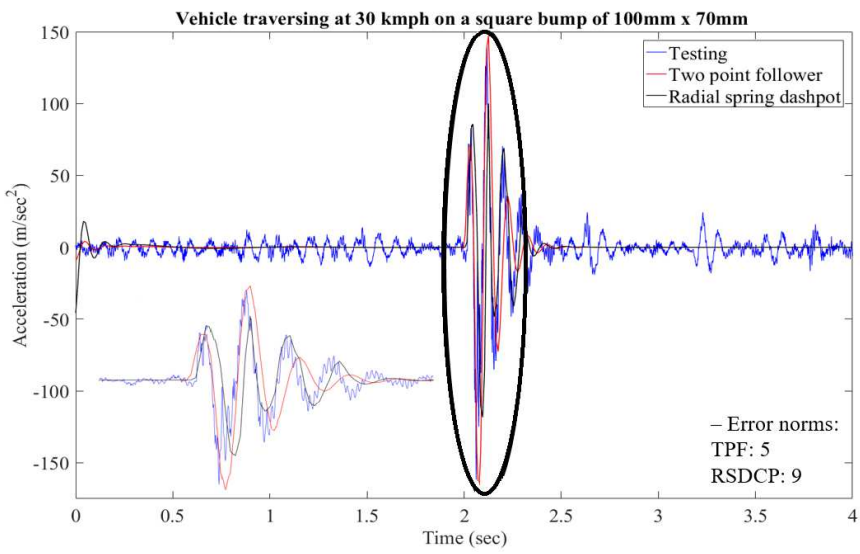

Fig. 7. Two point follower and radial spring-dashpot model responses against the measured axle responses. The zoomed portion shows that the two-point follower fits better to the test measurements as compared to the latter. The root mean square fitting error norms are also reported in the plot.

The reason for the mismatch is that the single point follower is the simplest model and does not consider any terrain filtering resulting in very high accelerations at wheel axles. In order to get a more realistic estimate of the wheel axle acceleration responses, a two-point follower and a radial spring-dashpot model was considered. ${ }^{1}$

It has been found that the best fit is obtained for the two-point follower as shown in Figure 7 and is further investigated by incorporating circular and sinusoidal basis functions.

For the two-point follower model, Zegelaar proposed a formulation to compute the virtual terrain (see Fig. 8).

This method necessarily computes an effective road surface which can be used to measure vehicle response over small constant height obstacles [31]. This method forms a good basis for model validation with respect to HIL vehicle test measurements.

Zegelaar's two-point follower technique requires a follower to go over a base curve (i.e., basis function). To begin with, a circular base curve was used which depicts the motion of the bottom-most point of a rigid tire rolling over an obstacle [32].

Consider a rigid tire rolling over a curb as shown in Figure 10a. For the rigid tire, the wheel center is lifted at a distance $L_{b}$ before the step when the wheel first hits the step (the step being at a longitudinal postion $X_{\text {step }}$ ). The shape of the axle position $Z_{\text {axle }}$ above road level versus position $X$ with $X$, varying from $X_{\text {step }}-L_{\mathrm{b}}$ to $X_{\text {step }}$, is found to be

$$
Z_{\text {axle }}=\sqrt{R^{2}-\left(X_{\text {step }}-X\right)^{2}}+h_{\text {step }}-R
$$

for wheel radius $R$, and step height $h_{\text {step. The length } L_{\mathrm{b}}}$ is given by

$$
L_{b}=\sqrt{R^{2}-\left(R-h_{\text {step }}\right)^{2}} .
$$

\footnotetext{
${ }^{1}$ Details of the radial spring-dashpot tire model are given in Appendix B.
}

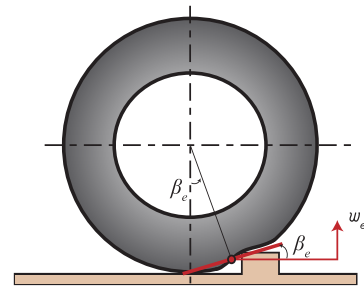

(a)

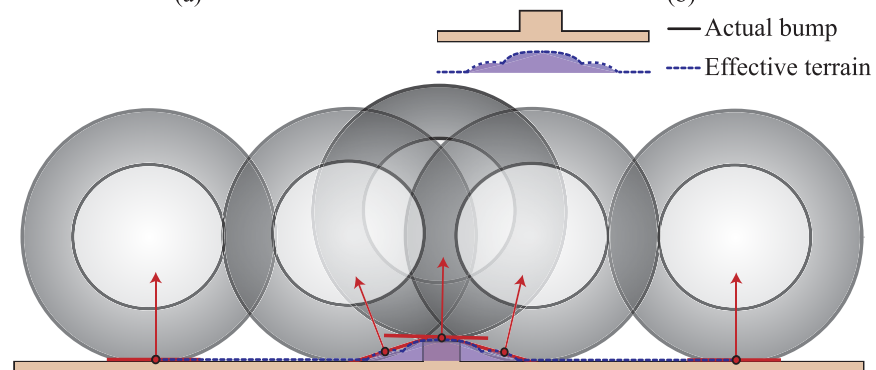

(c)
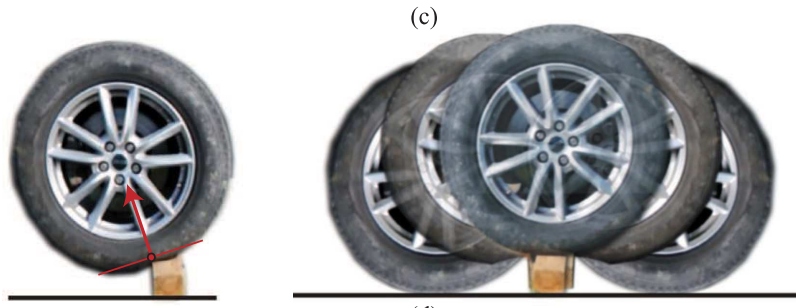

(d)

Fig. 8. Terrain filtering approach. (a) Effective plane height and angle; (b) dynamic tire load (components of the tire load normal to the effective plane $F_{N}$ : vertical $F_{z}$ and the horizontal $F_{b}$ also called as the bump resistance); (c) effective road terrain; (d) actual wheel deformation (left) and trajectory (right) over a bump during field trial.

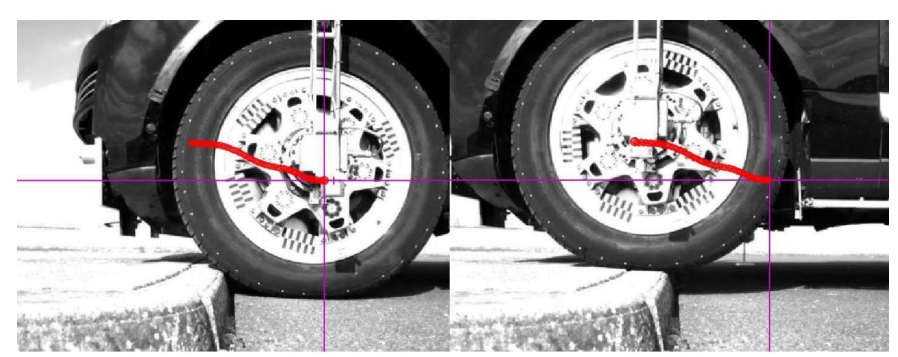

Fig. 9. Measured wheel centre trajectories of the front-left wheel of the vehicle going over the curb obstacle.

For the flexible wheel, however, the axle position appears to change according to a shifted superposition of two sinus-type curves [21]. This observation was initially made by Zegelaar and is used here to develop a pragmatic approach to derive the effective road surface. First the obstacle step input is used to derive a quarter sine wave basis function. This basis is defined for an $X$ interval of length $L_{b}$. Next it is shifted over a distance $L_{\mathrm{s}}$ in the direction of the step obstacle (see Fig. 10b). A two-point follower with length $L_{\mathrm{f}}$, is made to move along this shifted basis function. The midpoint of the follower describes the height $w_{e}$ of the effective road input and the 

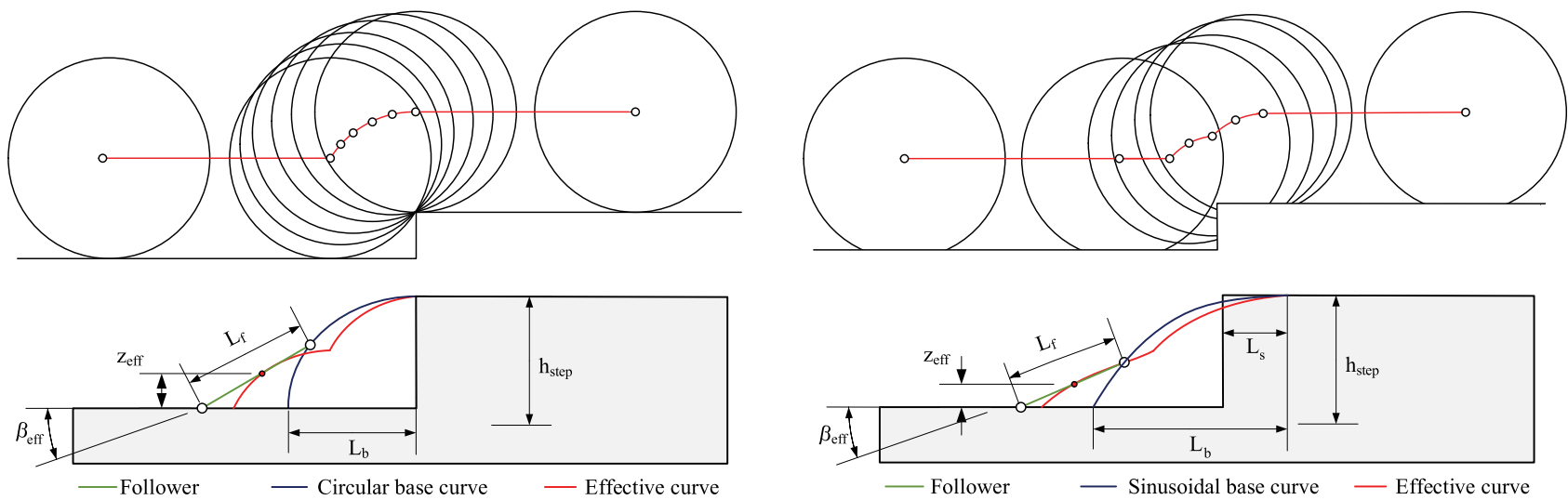

(a)

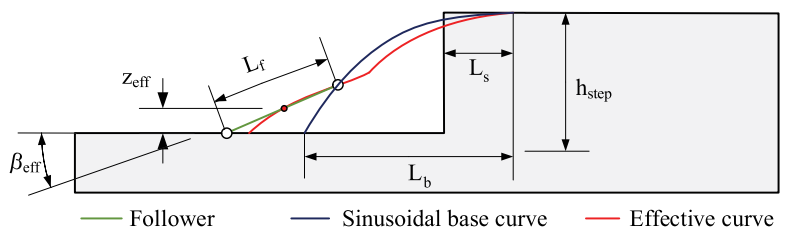

(b)

Fig. 10. Procedure to calculate effective terrain: (a) circular basis function; (b) sinusoidal basis function.

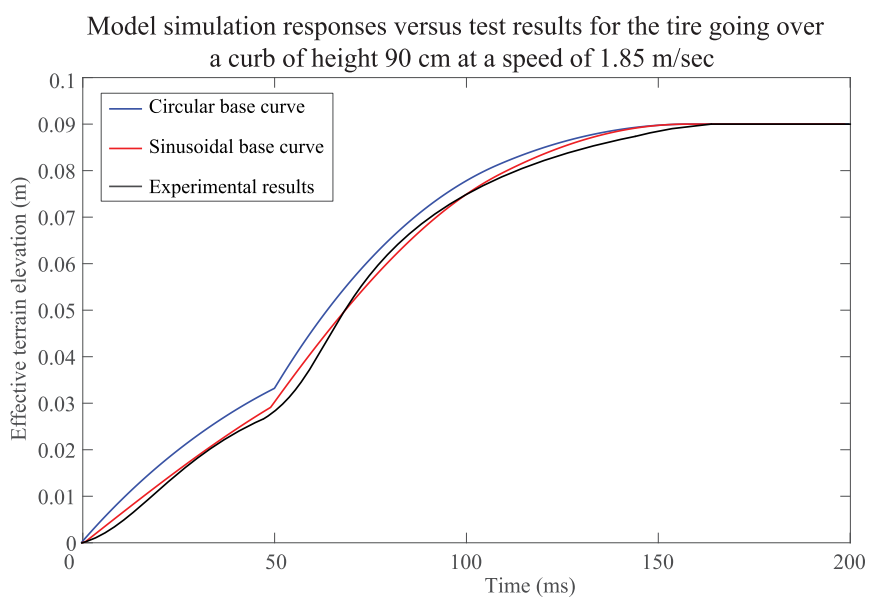

Fig. 11. Comparison of terrain enveloping models with test results.

angle describes the effective road slope $\beta_{e}$ (see Fig. 8a).

$$
\begin{aligned}
& w_{e}=\left(Z_{g 1}+Z_{g_{2}}\right) / 2 ; \\
& \beta_{e}=-\tan ^{-1}\left(\left(Z_{g_{1}}-Z_{g 2}\right) / l_{s}\right) .
\end{aligned}
$$

In equation (9), $Z_{g 1}, Z_{g 2}$ correspondings to the height of the front and rear points of the follower (for details see [33]).

To validate the computed terrains obtained by the twopoint follower model both with circular and sinusoidal basis functions, an experiment was performed to record the wheel motion going over a curb obstacle (see Fig. 9).

The wheel-centre trajectories were recorded for validating the simulation results obtained using the two-point follower model. For this purpose, the recorded test video is fed to the Tracker software [34]. The timeseries data of the vertical position of the front-left wheel centre is obtained from the inbulit image processing algorithm of the software.

The model fitting results are shown in Figure 11. The results show that the effective terrain obtained using a circular basis is non-smooth and the fit quality is inferior as compared to that with the sinusoidal basis. The twopoint follower with sinusoidal basis is thus used for the model development purposes.

To enhance the quality of the fit, a sinusoidal base curve has to be used as the tire is flexible in reality and the rigid tire approximation is not valid. The effective terrain obtained using sinusoidal basis strongly correlates that of the measured profile as shown in Figure 11.

\section{Tire modeling}

The role of tires is to provide the thrust necessary for the longitudinal motion of the vehicle. The friction force coefficient $\mu_{i}$ at the tire-road interface is a function of tire or wheel slip ratio $s_{i}$. According to the SAE definition, the longitudinal wheel slip ratio $s_{i}$ is defined as

$$
s_{i}=\frac{\omega_{i} R-V}{|V|} .
$$

In equation (10), $V$ is vehicle speed, $R$ is wheel radius and $\omega_{i}$ is wheel angular velocity. The slip ratio $s_{i}$ is a hyperbolic function of $V$ and is extremely sensitive to vehicle speed. A speed threshold $V_{t h}$ is artificially introduced to attenuate the sensitivity of $s_{i}$ otherwise the above slip definition will yield a very high value of slip at the start of simulation $\left(s_{i} \rightarrow \infty\right.$ as $\left.V \rightarrow 0\right)$ making the vehicle response unstable, resulting in simulation failure and making it unsuitable for real-time rig implementation. To solve this sigularity problem, a small threshold speed $V_{\text {th }}$ is routinely added to the denominator [35]. In the proposed model, a theshold speed of $0.2 \mathrm{~m} / \mathrm{sec}$ was used.

$$
s_{i}=\frac{\omega_{i} R-V}{|V|+V_{\mathrm{th}}} .
$$

However, this does not completely resolve the instability problem. The Magic Formula tire model uses a static definition of wheel slip which is extremely sensitive to the wheel's rotational speeds. These are measured using digital encoders on the rig whose sensitivity varies as wheel 


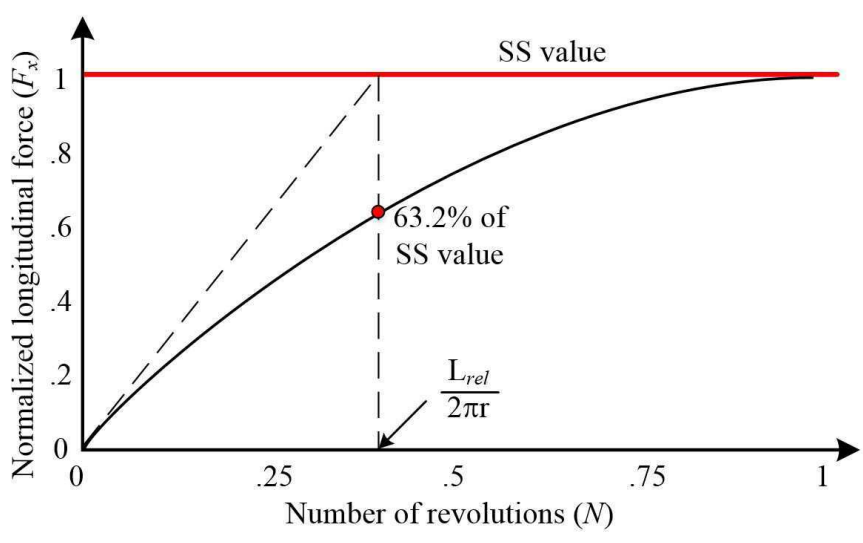

Fig. 12. Longitudinal force build-up: the concept of tire relaxation length and time constant [37].

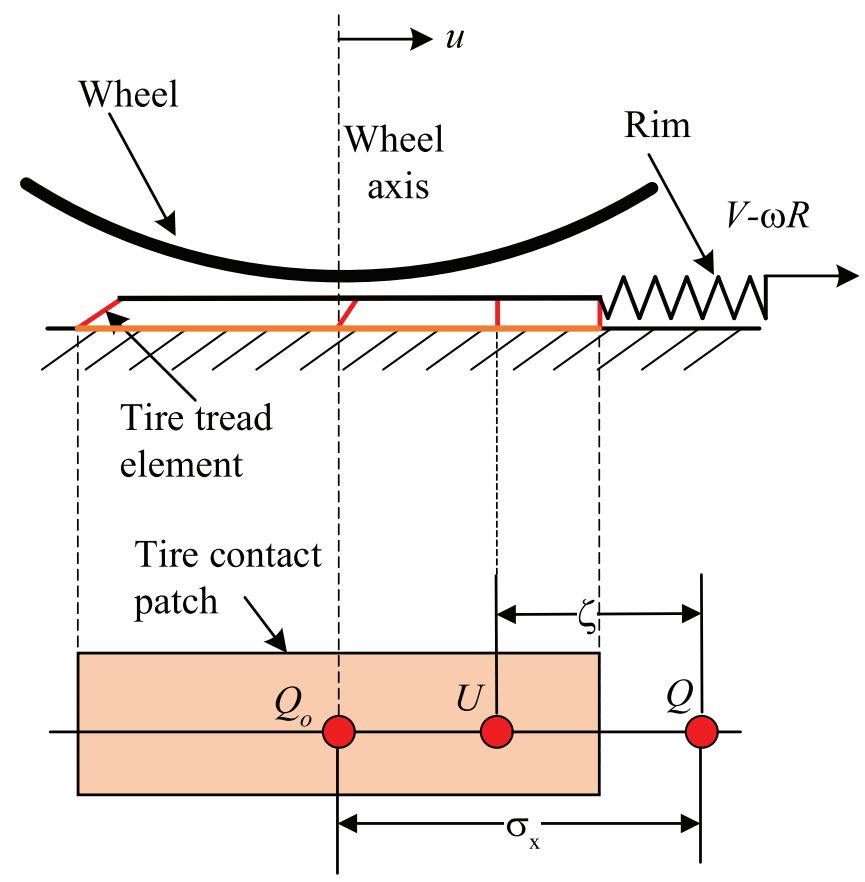

Fig. 13. Contact patch elemental deformation (adapted from [36]).

speed changes (low accuracy at low speeds). Clover et al. proposed the concept of longitudinal relaxation and time constant to define transient wheel slip $s_{i}^{\prime}$ to overcome stability issues and get a true estimation of the longitudinal wheel forces [36].

The main reason of introducing transient slip concept is to introduce a first order lag thereby the sensitivity of the longitudinal force on wheel slip is relaxed by means of a "relaxation length" (see Fig. 12).

Relaxation length or time constant concept is routinely used to characterize a first order lag in longitudinal force buildup with wheel slip. The tire relaxation length is that length of the tire contact patch which generates $63.2 \%$ of its steady state (constant) longitudinal force.

Consider the transient longitudinal slip model in Figure 13.

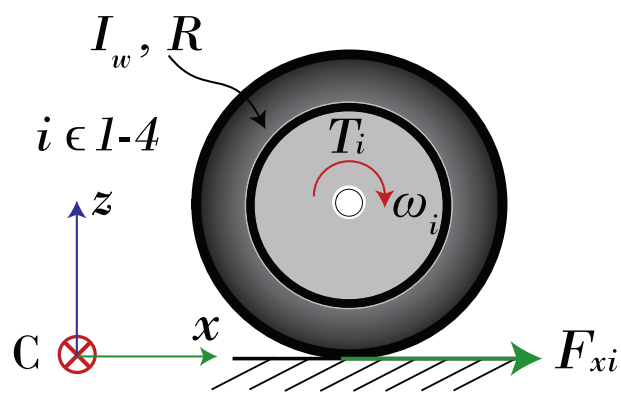

Fig. 14. Wheel angular dynamics.

The relaxation length $\sigma_{x}$ is the longitudinal deformation of a hypothetical point $Q_{o}$, currently at center of the undeformed contact patch to point $Q$. The distance from point $Q$ to the leading edge of the undeformed contact patch $U$ is $\zeta$ and it remains constant. $Q_{o}$ and $U$ are $\zeta$ and $\sigma_{x}$ behind $Q$ and their velocities are $V$ and $\omega R$ with respect to $Q$. The transient slip $s^{\prime}$ is defined as

$$
s_{i}^{\prime}=\frac{\left(\sigma_{x}-\zeta\right)}{\sigma_{x}}
$$

$\left(\sigma_{x}-\zeta\right)$ is the longitudinal deformation of $Q_{0}$. Differentiating $s^{\prime}$ with respect to time by tracking points $Q_{0}$ and $U$ yields

$$
\frac{d s_{i}^{\prime}}{d t}+\frac{V}{\sigma_{x}} s_{i}^{\prime}=\frac{V-r \omega}{\sigma_{x}} .
$$

In equation (13), the relaxation length $\sigma_{x}$ is given by

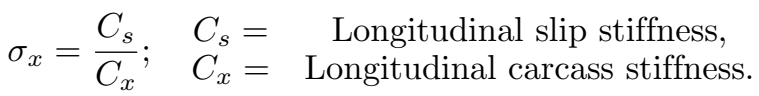

For the tires used herein, the relaxation length was found to be $90 \mathrm{~mm}$. Solving Equation (13) gives the value of transient slip $s^{\prime}$ which is then used in the Magic Formula tire model to obtain the longitudinal force coefficient [38].

$$
\mu_{i}=D \sin \left(C \tan ^{-1}\left(B \cdot s_{i}^{\prime}\right)\right)
$$

In the above equation, $B$ is the stiffness factor (controls the slope of friction coefficient versus slip curve at origin), $C$ is the shape factor (limits the range of arguments in the sine function) and $D$ is the maximum value of friction coefficient. The product BCD gives the slope and corresponds to initial longitudinal slip stiffness. Using $\mu_{i}$ the longitudinal tire force $F_{x i}$ is obtained as

$$
F_{x i}=\mu_{i} F_{z i}
$$

Each wheel is subjected to drive torque $T_{i}$ and the resistive frictional torque due to force $F_{x i}$ calculated above. The wheel angular dynamics is shown in Figure 14.

$$
I_{w} \dot{\omega}_{i}=T_{i}-F_{x i} R ; \quad T_{i} \text { is wheel torque. }
$$




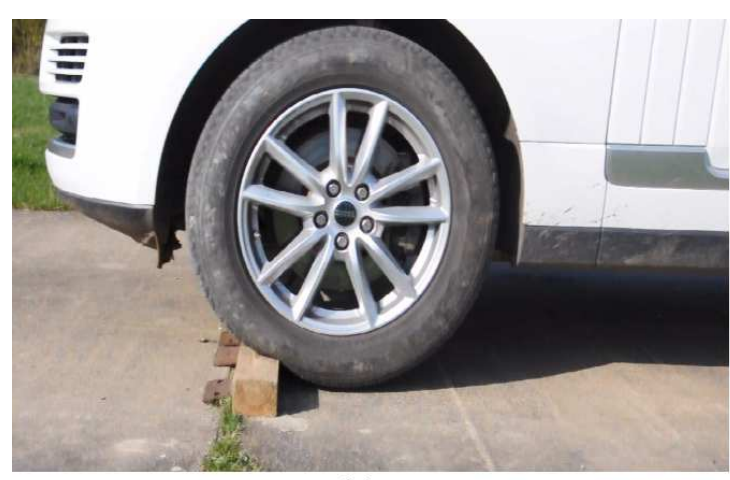

(a)

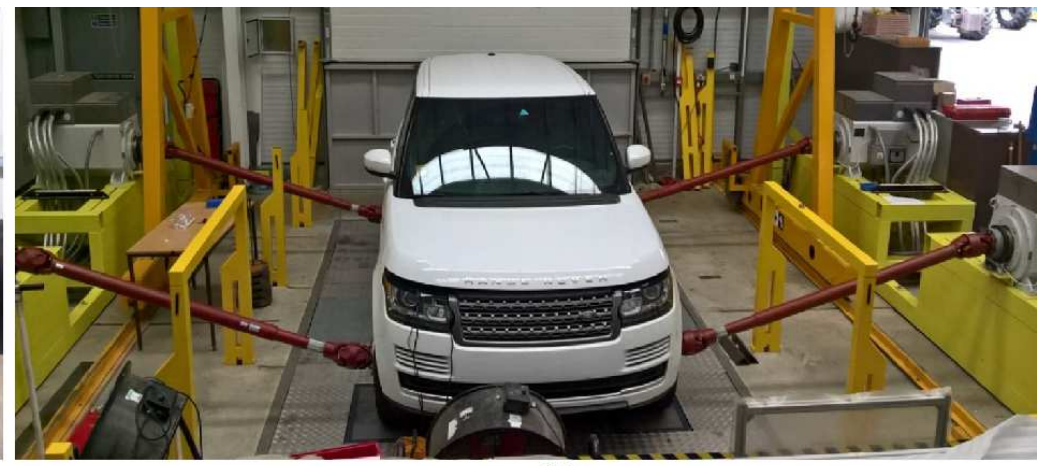

(b)

Fig. 15. (a) Vehicle going over a rectangular bump. (b) Vehicle mounted on the ORES test-rig.

\section{Driveline model}

The wheel torque $T_{i}$ is obtained from the driveline model. It necessarily consists of an engine map (made available by Jaguar Land Rover), speed-dependent final gear ratio (automatic transmission) and an effective driveline inertia at the wheels. This driveline model is used for offline field tests. For real-time rig testing, the actual driveline of the vehicle replaces driveline model as discussed in Section 7.

\section{Offline and rig models}

As described previously, two versions of vehicle models were developed; one for field tests with in-built driveline model; and the second one for the rig testing. In the rig model, the actual vehicle driveline replaces the driveline model and the resistive frictional torques are applied by the dynos instead of tires. The offline model is used for the validation of field testing results and is developed in MATLAB/Simulink environment while the online model is used for rig testing and it has to work in real-time so it has been loaded in the Speedgoat target PC using the Simulink-RT interface as discussed earlier in Section 2. The modeling details of the offline and online rig models are discussed in Appendix A.

\section{Results: field vs rig testing}

In order to validate the vehicle model, both field trials and rig trials were performed (see Fig. 15a and b). These validation trials were necessary to ensure that the model is able to accurately capture the essential dynamic characteristics of the vehicle going over a discrete bump. Different bump shapes like step (or curb), square, rectangular and trapezoidal were chosen and the vehicle was driven at a range of speeds over these bumps. For model validation, the vertical acceleration of the front-left wheel axle was recorded using an accelerometer and four wheel speeds and torques were recorded using speed encoders and wheel torque transducer as shown in Figure 16.

One such test scenario of the vehicle traversing a rectangular bump $(40 \times 70 \mathrm{~mm})$ at low speed (around $7 \mathrm{kmph})$

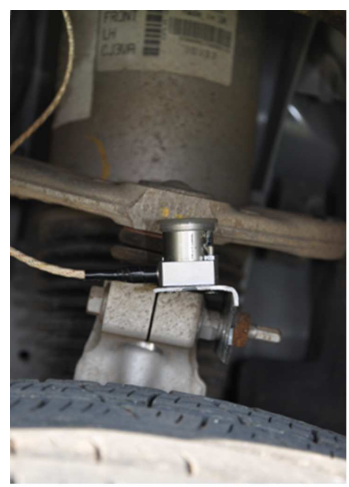

(a)

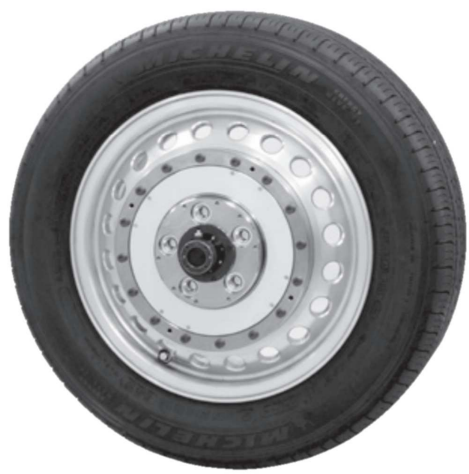

(b)
Fig. 16. (a) Accelerometer on axle. (b) Wheel torque transducer.

is chosen to demonstarte both the offline model simulation and viability of the real-time model on the rig. Same vehicle speeds were maintained in the offline simulation by appropriately choosing the drive torque demand in driveline model. While driving the vehicle on rig, the driver carefully monitors speed encoder digital display signal to replicate the field test scenario.

The offline simulation results (namely front-left axle acceleration, four wheel vertical loads, speeds and torques) are shown in Figure 17.

The simulation results are then compared with rig and field test measurements in Figure 18. A brief discussion of these results is presented below.

The vehicle speed profile in both field and rig tests is identical to that of the simulation. This justifies that same test conditions were maintained during both simulation and rig testing. During the trials the bump was located only in the left half of the vehicle and as a result only front and rear-left wheels encountered the bump. The wheel's vertical acceleration has two distinct peaks in the response. The larger peak is observed while the front wheel encounters the bump and the smaller peak corresponds to the rear left wheel bump encounter. As the wheel hits the bump the vertical acceleration increases and correspondingly the normal load on the wheel increases too. The wheel loads are an output of the real-time model on the rig but they are not available during field trial as it 


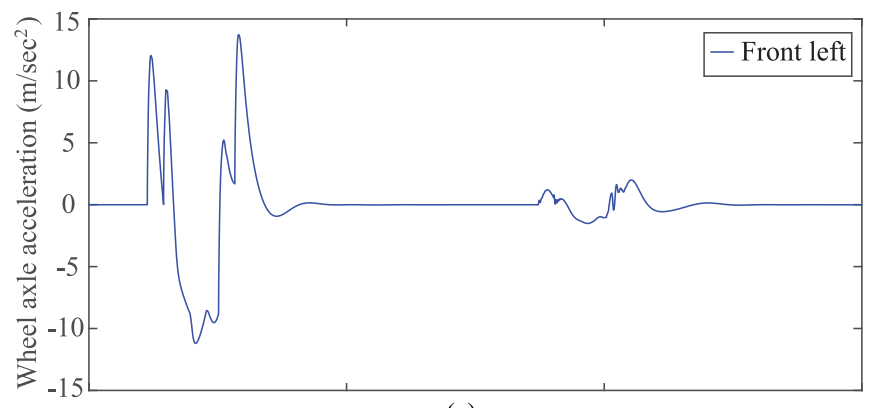

(a)

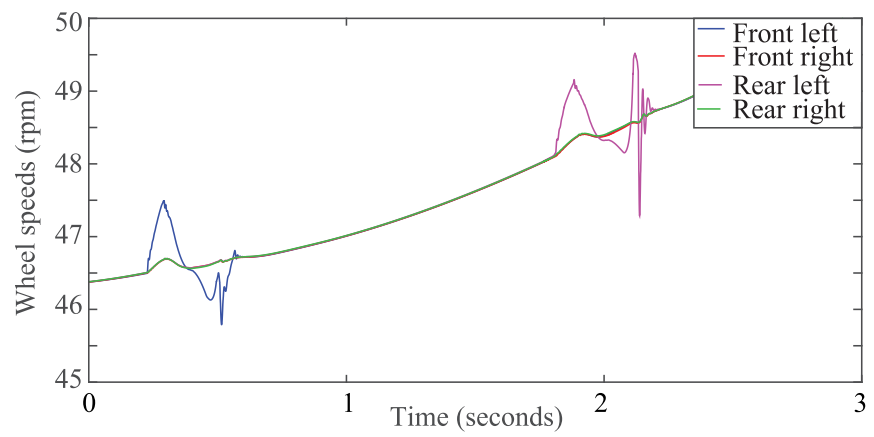

(c)

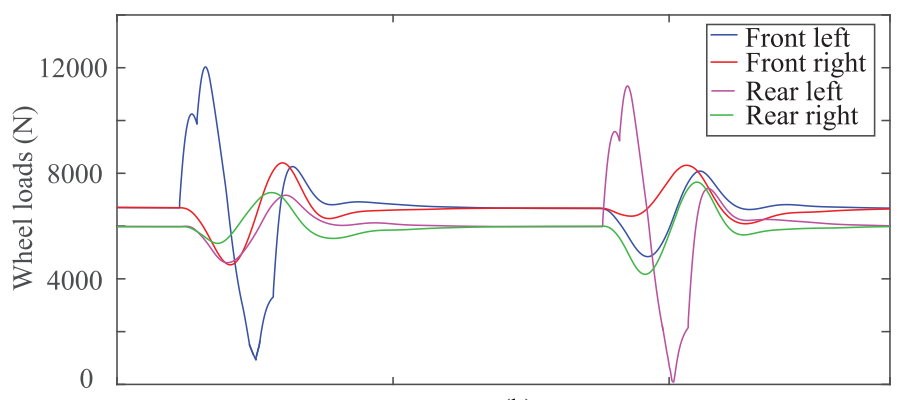

(b)

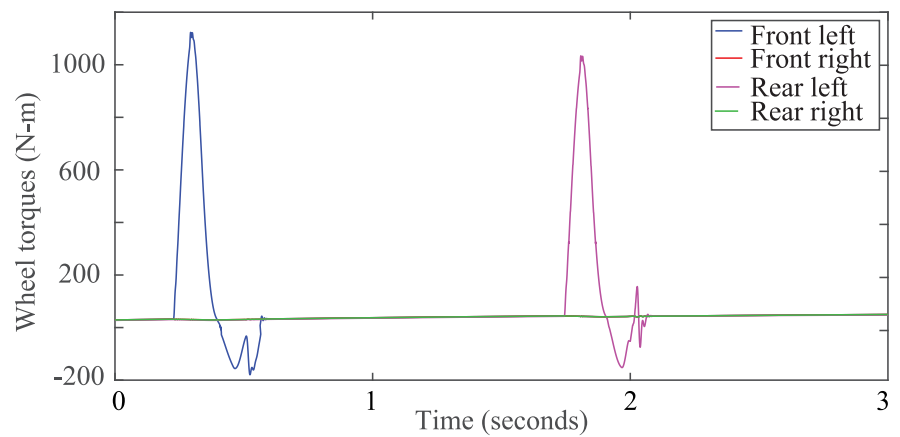

(d)

Fig. 17. Simulation results: (a) front-left axle acceleration; (b) wheel normal loads; (c) wheel speeds; (d) wheel torques.
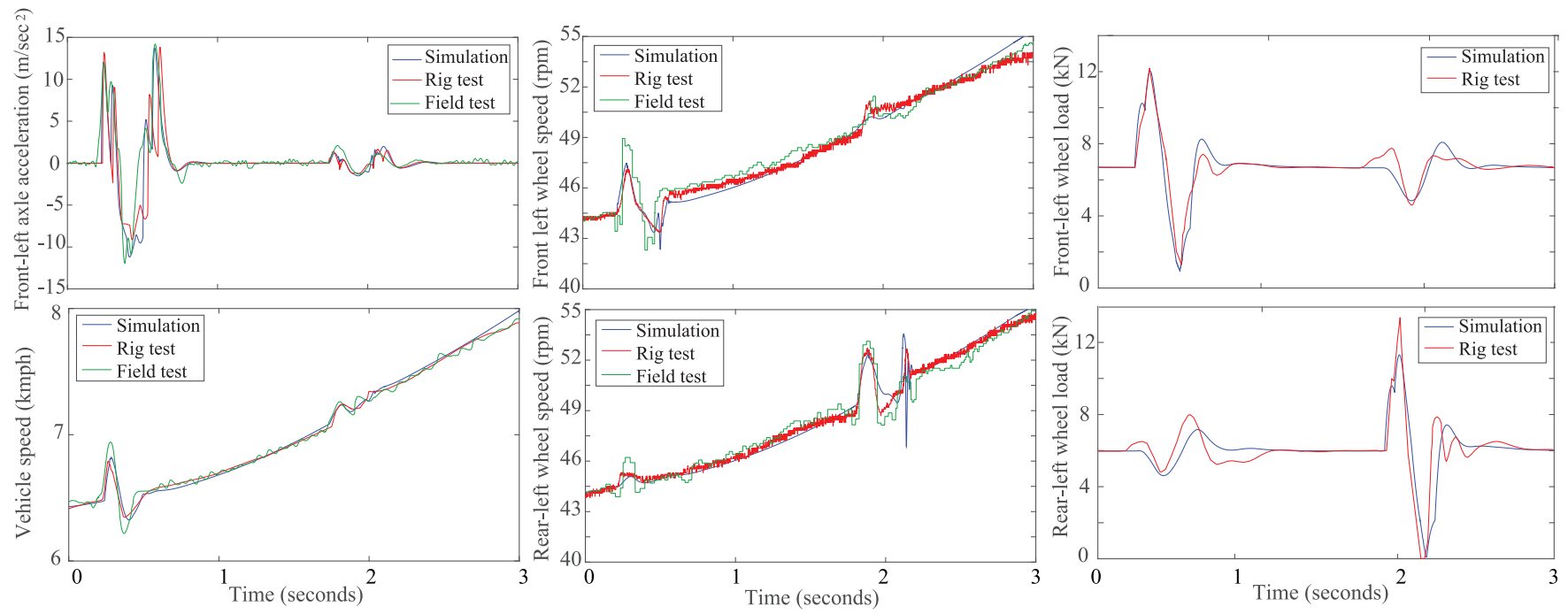

Fig. 18. Simulation versus test results.

was not possible to record the normal loads on the wheel (wheel suspension-load/travel measurement was not measured) during the field trial. A larger value of wheel torque is required to negotiate the obstacle and as a result the wheel's angular speeds increases as well. For the rearleft wheel, the first peak in both wheel speed and loads is smaller as compared to the second peak which corresponds to the effect of front-left bump on the rear-left wheel.

The rig-test wheel acceleration responses have a close agreement with that of the field test responses. During the rig test, the normal load on the wheel momentarily becomes zero which shows that the wheel looses contact for a small period of time, while this was not observed during the field tests. This is one potential reason for the mismatch. However the peak heights, width and timings closely match in both rig model and the offline model which demonstrates the suitability of proposed modelling approach.

\section{Conclusions}

The aim of this paper is to replicate off-road field testing conditions in a lab-based testrig. The vehicle 
model was developed and interfaced with a real-time hardware-in-the-loop test rig. The model was developed in MATLAB/Simulink and different kinds of terrain filtering approaches were explored. The two-point follower technique with sinusoidal enveloping was found to be the best technique and was used to define an equivalent terrain profiles. The terrain model is interfaced with a detailed 7-dof ride model, a longitudinal dynamic vehicle model and a Magic Formula based tire model along with modified slip (transient) definition to overcome the stability issues. These individual subsystem models are interfaced in a logical fashion to build two versions of the complete vehicle model; one for the field tests with in-built driveline and the other for rig testing. These models were successfully validated against field test and rig simulation results. The evaluation of the equation set in real-time was easily achieved in the target hardware deployed.

\section{Future work}

In the future, the two-point follower model would be extended to include elliptical enveloping for use over a more generic terrain made of discrete square bumps. So far in this paper, single-point, two-point follower and radial spring tire model are considered. More sophisticated tire models like radial-interrelated spring model can be explored. In both rig runs and field trials, the vehicle is driven over small bumps at low speed where the wheel does not completely detach from the ground. At high speeds, the wheel lifts off the ground and the model needs to be extended to simulate wheel detachment. The vehicle suspensions were modelled using linear Kelvin-Voigt model, for larger bump excitations suspension nonlinearity creeps in and suspension nonlinearity needs to be considered. Other complicated scenarios like rock crawl, terrain with variable friction, driving uphill and downhill with variable gradient will also be considered.

\section{Appendix A: Offline and online rig models}

As discussed in Section 7, two versions of vehicle models were developed corresponding to that of field (offline) and rig (RT-online) testing. The offline model is shown in Figure A.1.

It consists of a terrain model, full car ride model, a bump resistance model to calculate the resistance offered by the bumps to the wheels, a longitudinal dynamics model along with a longitudinal slip model to calculate the wheel resistive forces and eventually a driveline model to replicate the engine and the powertrain which generates the torque request from the throttle pedal input from the driver. Each of these models are implemented in a modular way using Simulink suybsystems.

For the rig model, the driveline Simulink subsystem does not exists. The torque request is generated directly by the engine and driveline in the physical vehicle that is mounted on the ORES rig. The four wheel speeds were measured from the rig (see red subsystem in Fig. A.1) and used as inputs to the longitudinal dynamics block at the location indicated by the red circle. The resistive torques calculated by the longitudinal dynamics block (shown by the blue circle) are used as inputs to the four dynos as shown by the blue subsystem. These dynos are eventually used to apply these resistive torques on the wheels using a shaft-hub coupling as shown in Figure $15 \mathrm{~b}$.

\section{Appendix B: Radial spring dashpot tire-terrian enveloping}

For the radial spring-dashpot model, the tire-road interaction in the vertical plane is represented by continuously

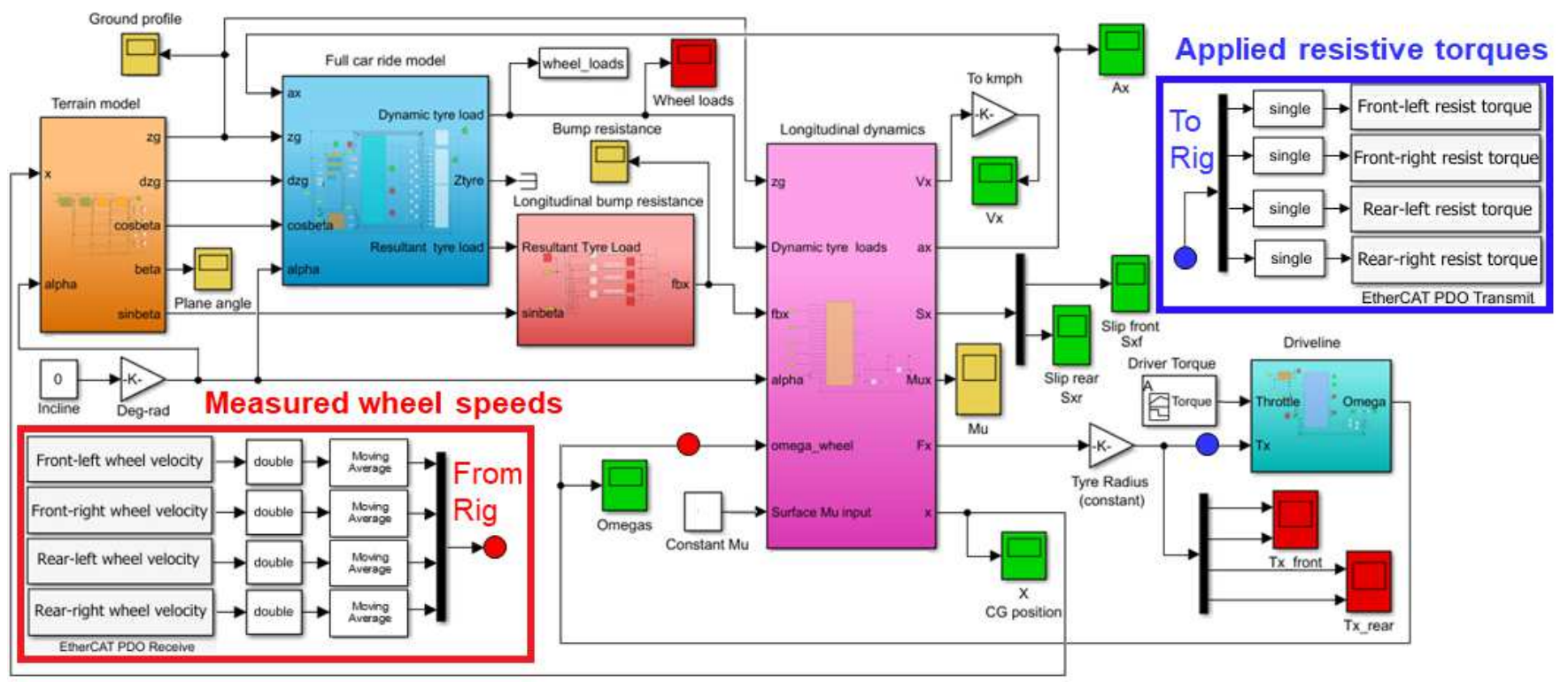

Fig. A.1. Offline and rig models developed in MATLAB/Simulink platform. 


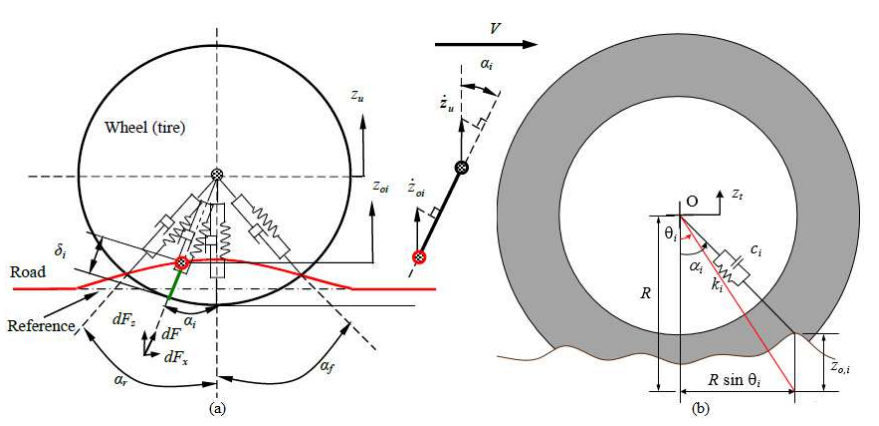

Fig. B.1. (a) Radial spring-dashpot tire-terrain model (adapted from [39]). (b) Individual spring-dashpot element.

distributed radial spring-damping elements symmetric to the wheel center. The tire deforms only in radial direction while circumferential shear deformation is neglected (see Fig. B.1).

The radially distributed linear spring-dashpot elements with stiffness $k_{t, j}$ and damping coefficients $c_{t, j}$ are defined as the magnitude of force required to produce unit radial deformation of the spring and relative speed change within unit angle, respectively. The elemental spring stiffness, damping coefficient and spring and damping force is given by (for details see [40]).

$$
F_{k}=\sum_{j=1}^{n_{r}} k_{t, j}\left[R-\sqrt{\left(R \sin \theta_{j}\right)^{2}+\left(R+z_{t}-z_{0 . j}\right)^{2}}\right] \cos \alpha_{j}
$$$$
F_{c}=\sum_{j=1}^{n_{p}} c_{t, j}\left(\dot{z}_{0, j}-\dot{z}_{t}\right) \cos \alpha_{j}
$$

$k_{t, j}=\frac{k_{t}}{n_{p}} ; \quad c_{t, j}=\frac{c_{t}}{n_{p}}$

For the proposed modeling approach eight linear springdashpot elements were considered in the contact patch regime.

Acknowledgements. This work was done under "Eastnor Loop Simulation Study" project at Cranfield University. The aim of the project was to develop hardware-in-the-loop techniques using production vehicles from Jaguar Landrover (JLR) on the Off-Road Environment Simulator, a 4 wheel highly transient transmission dynamometer built at the offroad engineering facility in Cranfield. The author wishes to thank the offroad facility laboratory staff for their support in the field testing work; Jamie Foster from JLR for providing the vehicle data; Kim Blackburn for endless technical discussion and support during the rig testing and vehicle instrumentation; and Antony Allen for proof-reading the manuscript.

\section{References}

[1] M. Bartram, G. Mavros, S. Biggs, A study on the effect of road friction on driveline vibrations. Proc. Inst. Mech. Eng. K 224, 321-340 (2010)
[2] M. Ahmadian and W. Huang, A numerical evaluation of the suspension and driveline dynamic coupling in heavy trucks. SAE Trans. 560-569 (2004)

[3] H. Kanchwala, A.S. Trigell, Vehicle handling control of an electric vehicle using active torque distribution and rear wheel steering. Int. J. Vehicle Des. 74, 319-345 (2017)

[4] H. Kanchwala, J. Wideberg, Pitch reduction and traction enhancement of an EV by real-time brake biasing and inwheel motor torque control. Int. J. Veh. Syst. Model. Test. 11, 165-192 (2016)

[5] Ph. Couderc, J. Callenaere, J. Der Hagopian, G. Ferraris, A. Kassai, Y. Borjesson, L. Verdillon, S. Gaimard, Vehicle driveline dynamic behaviour: experimentation and simulation. J. Sound Vib. 218, 133-157 (1998)

[6] A. Sorniotti, Driveline modeling, experimental validation and evaluation of the influence of the different parameters on the overall system dynamics. Technical report, SAE Technical Paper (2008)

[7] J.Y. Wong, Theory of ground vehicles. John Wiley \& Sons (2008)

[8] G. Mavros, Contact mechanics of tyre-road interactions and its role in vehicle shuffle. In Tribology and Dynamics of Engine and Powertrain (Elsevier, 2010), pp. 703-734

[9] W. Li, X.H. Shi, D. Guo, P. Yi, A test technology of a vehicle driveline test bench with electric drive dynamometer for dynamic emulation. Technical report, SAE Technical Paper (2015)

[10] AWD, Range Rover Sport. https://www.landrover.co.uk/ vehicles/range-rover-sport/index.html

[11] H. Pacejka, Tire and vehicle dynamics (Elsevier, 2005)

[12] G.N.B. Hathron, The Development Of An Off-Road Environment Simulator (ORES). PhD Thesis. Cranfield University, 2011

[13] R.D. Nine, N.N. Clark, J.J. Daley, C.M. Atkinson, Development of a heavy-duty chassis dynamometer driving route. Proc. Inst. Mech. Eng. D 213, 561-574 (1999)

[14] F.C. Teng, Real-time control using matlab simulink. In Smc 2000 conference proceedings. 2000 IEEE international conference on systems, man and cybernetics. 'cybernetics evolving to systems, humans, organizations, and their complex interactions' (cat. no. 0, Vol. 4 (IEEE, 2000), pp. 2697-2702

[15] H. Kanchwala, I. Bezerra Viana, N. Aouf, Cooperative path-planning and tracking controller evaluation using vehicle models of varying complexities. Proc. Inst. Mech. Eng. C 0954406220945468 (2020)

[16] A. Soltani, A. Goodarzi, M.H. Shojaeefard, K. Saeedi, Optimizing tire vertical stiffness based on ride, handling, performance, and fuel consumption criteria. J. Dyn. Syst. Measur. Control 137 (2015)

[17] H. Kanchwala, Studies in simplified dynamic modeling and characterization of vehicle suspensions. PhD Thesis, Indian Institute of Technology, Kanpur. (2017)

[18] L. Li, C. Sandu, Modeling and simulation of $2 \mathrm{~d}$ arma terrain models for vehicle dynamics applications. SAE Trans. 1-9 (2007)

[19] H. Kanchwala, A. Chatterjee, Adams model validation for an all-terrain vehicle using test track data. Adv. Mech. Eng. 11, $1687814019859784(2019)$

[20] K.M. Captain, A.B. Boghani, D.N. Wormley, Analytical tire models for dynamic vehicle simulation. Vehicle Syst. Dyn. 8, 1-32 (1979) 
[21] P.W.A. Zegelaar, H.B. Pacejka, The in-plane dynamics of tyres on uneven roads. Vehicle Syst. Dyn. 25, 714-730 (1996)

[22] A.J.C. Schmeitz, A semi-empirical three-dimensional model of the pneumatic tyre rolling over arbitrarily uneven road surfaces (2004)

[23] J.R. Kilner, Pneumatic tire model for aircraft simulation. J. Aircraft 19, 851-857 (1982)

[24] K. Guo, Tire roller contact model for simulation of vehicle vibration input. Technical report, SAE Technical Paper, 1993

[25] J. Kisilowski, Z. Lozia, Modelling and simulating the braking process of automotive vehicle on uneven surface. Vehicle Syst. Dyn. 15, 250-263 (1986)

[26] J.M. Badalamenti, G.R. Doyle Jr., Radial-interradial spring tire models. J. Vib. Acoust. Stress Reliab. Des. 110, 70-75 (1988)

[27] B.G. Kao, M. Muthukrishnan, Tire transient analysis with an explicit finite element program. Tire Sci. Technol. 25, 230-244 (1997)

[28] A.J.C. Schmeitz, J.P. Pauwelussen, An efficient dynamic ride and handling tyre model for arbitrary road unevennesses. In Reifen, Fahrwerk, Fahrbahn: Tagung Hannover, 18-19 Oktober 2001. VDI-Verlag (2001) 173-199

[29] M. Eichler, A ride comfort tyre model for vibration analysis in full vehicle simulations. Vehicle Syst. Dyn. 27, 109-122 (1997)

[30] I.B. Viana, H. Kanchwala, K. Ahiska, N. Aouf, A comparison of trajectory planning and control frameworks for cooperative autonomous driving. J. Dyn. Syst. Measur. Control (2021)

[31] P.W.A. Zegelaar, The dynamic response of tyres to brake torque variations and road unevennesses (1998)

[32] J. Pauwelussen, Essentials of vehicle dynamics (Butterworth-Heinemann, 2014)

[33] J.S. Dhillon, Vehicle dynamics modelling for an off-road high performance application using a multibody systems approach (2016)

[34] Tracker. Open Source video analysis and modeling tool. https://physlets.org/tracker/

[35] S. Jung, T.Y. Kim, W.S. Yoo, Advanced slip ratio for ensuring numerical stability of low-speed driving simulation. Part I: Longitudinal slip ratio. Proc. Inst. Mech. Eng. D 233, 2000-2006 (2019)

[36] C.L. Clover, J.E. Bernard, Longitudinal tire dynamics. Vehicle Syst. Dyn. 29, 231-260 (1998)

[37] P. Fancher, J. Bernard, C. Clover, C. Winkler, Representing truck tire characteristics in simulations of braking and braking-in-a-turn maneuvers. Vehicle Syst. Dyn. 27, 207-220 (1997)

[38] H. Kanchwala, J. Wideberg, C. Bordons Alba, D. Marcos, Control of an independent 4WD electric vehicle by DYC method. Int. J. Veh. Syst. Model. Test 10, 168-184 (2015)

[39] J.J. Zhu, Study of vehicle dynamics with planar suspension systems (pss) (2011)

[40] Y.C. Chang, T.T. Fu, A useful tire model for ATV ride performance on rough roads. Technical report, SAE Technical Paper (2010)

Cite this article as: H. Kanchwala, ORES: a chassis dynamometer for off-road vehicles. Mechanics \& Industry 22, 6 (2021) 